Voix et Images

voixetimages

\title{
La démarche sociocritique au Québec
}

\section{André Belleau}

Volume 8, numéro 2, hiver 1983

Marie-Claire Blais

URI : https://id.erudit.org/iderudit/200384ar

DOI : https://doi.org/10.7202/200384ar

Aller au sommaire du numéro

\section{Éditeur(s)}

Les Presses de l’Université du Québec

\section{ISSN}

0318-9201 (imprimé)

1705-933X (numérique)

Découvrir la revue

\section{Citer cet article}

Belleau, A. (1983). La démarche sociocritique au Québec. Voix et Images, 8(2), 299-310. https://doi.org/10.7202/200384ar d'utilisation que vous pouvez consulter en ligne.

https://apropos.erudit.org/fr/usagers/politique-dutilisation/ 


\title{
La démarche sociocritique au Québec
}

\author{
par André Belleau. Université du Québec à Montréal
}

S'il est tout à fait légitime de parler de la "méthode sociocritique», on a bien du mal, en revanche, à parler de cette méthode "méthodiquement ". Certes, rien n'empêche d'affirmer doctement: la méthode sociocritique est l'ensemble des movens conceptuels, analytiques et discursifs mis en œuvre pour l'étude des déterminations et de la signification sociales des textes littéraires. La difficulté commence lorsqu'on se met à regarder les choses de plus près, nommément divers discours critiques. Voilà le lieu, semble-t-ii, d'une șorte de fonctionnement ou plutôt d'évidence sur pièces, par à-coups: si le livre ou l'article emporte l'adhésion, donne l'impression que * ca marche*, on dira: voila de la sociocritique. Je ne dis pas qu'il n'y a pas de méthode. Je défie tout simplement quiconque de me décrire en quelques pages la MÉTHODE d'Auerbach dans Mimésis' ${ }^{1}$, de Lukács dans la Théorie du roman ${ }^{2}$ (sa méthode, pas ses fondements philosophiques!), d'Adorno dans les Noten zur Literatur ${ }^{3}$, de Bakhtine dans son Rabelais ${ }^{4}$. Et l'on étonnera peut-être plusieurs lecteurs en disant que Barthes fut aussi un grand sociocritique (ce qui traduit tout simplement la non-pertinence croissante de la répartition actuelle du champ entre la sémiologie. la psychanalyse littéraire, et ce qu'on. appelle souvent l'approche socio-historiquel.

II ne s'agit nullement de proposer (et justifier) a posteriori une sorte d'empirisme fatal dû à l'état précaire de la théorie. Aucun des notables. critiques que je viens de nommer n'est un empiriste. Ce qu'il faut garder a l'esprit, c'est que la socialité est une dimension tellement primordiale de tout discours qu'il convient d'envisager la sociocritique moins comme une méthode reconnue que comme un vaste domaine regroupant l'ensemble des recherches visant principalement ou accessoirement a rapporter les systèmes observés dans les œuvres au discours social ambiant, ou aux idéologies, ou a l'institution littéraire, ou encore a la structure de la société, à la position d'un groupe, d'une classe, à la situation économique, etc. S'il parait abusif de ramener toutes ces entreprises à une méthode (ou même à deux ou trois), on est quand même en droit d'y constater des tendances assez générales.

Notons d'abord l'existence au Québec d'une "sociocritique" traditionnelle, une sorte de discours sociologisant général pratiqué depuis toujours par les journalistes, les écrivains, les professeurs, qui tend à considérer chaque cuvre importante parue comme un moment réussi ou non d'une évolution littéraire inséparable de l'évolution nationale et politique. On est 
enclin à parler ici d'une critique quasi "spontanée " tant elle s'avère à la fois largement répandue et tout a fait inconsciente de son caractère moralisateur. Elle est peut-être aussi la plus facile: on a souvent tendance à se rabattre sur le social lorsqu'il y a pauvreté ou pénurie du signifiant. Je me souviens qu'à la parution du Journal dénoué de Fernand Ouellette ${ }^{5}$ (réussite incontestable sur le plan littérairel, un critique déclara à la radio que l'espèce de libération personnelle dont Ouellette faisait état dans son livre signifiait sans aucun doute le progrès mème du Québec. II ne lui était pas venu à l'esprit: $1{ }^{\circ}$ que la valeur très grande du Journal dénoué ne repose pas sur les événements de la vie de son auteur mais sur la manière dont celui-ci les relate; $2 \%$ que le fait pour une personne d'être alibérée (si tant est que cela veuille dire quelque chose) ne la rend pas nécessairement meilleur écrivain; $3^{\circ}$ ) que la série littéraire et la série politique, pour reprendre les termes des formalistes russes. quoiqu' ayant des rapports entre elles, ne sont nullement isochrones. L'exemple fait voir cette sociologisation (se donnant comme anaturelle») dans son fonctionnement positif. Car elle opère aussi négativement. Cela consiste, notamment pour les cuvre d'avant mil neuf cent soixante, à substituer une négativité à une positivité. Selon elle, la littérature québécoise a été empèchée. mutilée par la toute-puissance du clergé, les interdits de toutes espèces, etc. Cette interprétation n'a que le défaut d'être trop simple. On songe au mot de Pierre Zima : a La monosémie et le systeme du Parti unique sont homologues. $\theta$ Ce qu'il restait à expliquer, c'était l'essentiel: pourquoi n'avons-nous pas ou alors une grande poésie catholique, un grand roman chrétien? Après tout, le siécle d'or de la littérature espagnole fut aussi celui de la sainte Inquisition. La vision sociologisante traditionnelle a souvent médiatisé l'insuffisance sociale générale par le udrame spirituel \ de l'auteur. lui-mème attribuable da la société. C'est ce qu'a fait Jean Le Moyne dans un texte fameux: « Saint-Denys Garneau, témoin de son temps»?. Jean Le Moyne ne pouvait pas s'aviser que la question importante, du point de vue d'un discours critique sérieux, n'est pas le non-accomplissement de l'homme mais bien l'accomplissement (dans quelle mesure?) de son couvre et que chez un écrivain, on doit lire le malheur du temps dans le bonheur des mots. En termes plus techniques: I'inscription de la société dans un texte littéraire n'est repérable et analysable que dans le degré et la manière dont ce dernier parvient à subsumer les codes qu'il signale. Mais gardons-nous d'afficher envers nos devanciers un quelconque air de supériorité: le discours critique, positif ou négatif, de l'émancipation nationale-artistique demeure une tentation constante de la conscience littéraire québécoise. J'en donne comme preuve l'ouvrage d'Axel Maugey, Poésie et société au Québec (1937-1970). ${ }^{B}$ Tant que le problème national québécois n'aura pas été réglé, les divers discours critiques éprouveront de la difficulté à justifier leur autonomie théorique et à accréditer des pratiques qui leur sont propres.

Si l'on excepte le rayonnement toujours important (et diffus) du Sartre de Qu'est-ce que la littérature? et de Questions de méthode ${ }^{9}$, la grande influence pendant les années soixante fut celle de Lucien Goldmann. Contrairement à plusieurs réussites notables de la sociocritique, le Dieu caché $^{10}$, qui fut et demeure un grand livre, propose expressément et systé- 
matıquement une méthode. En fait, on constate peu de traces de I'utilisation de cette méthode par des critiques québécois, sauf, si je ne $m$ 'abuse. dans Pierre Perrault ou un cinéma national du sociologue Michel Brülé. "II semble que le rôle de Goldmann ait été surtout celui d'un instigateur, d'un éveilleur, du fait notamment de Pour une sociologie du roman ${ }^{12}$, ouvrage pourtant très inférieur au Dieu caché: la sociocritique, renonçant à toute médiation, s'y dégrade en sociologisme vulgaire. Mais Goldmann nous a montré qu'une étude sociale du texte pouvait et devait ètre tentée. On retrouve dans JeanCharles Falardeau le concept goldmannien de "vision du monde" mais sûrement pas la méthode goldmannienne elle-même.

La difficulté chez Goldmann résidait dans l'exclusion du plan de l'expression, en fait des traits les plus distinctifs du discours littéraire. "Expliquer: (le vocabulaire goldmannien garde une coloration positiviste) les tragédies de Racine par la structure dune avision tragiquen commune a Phèdre et au groupe social, c'était se limiter à la forme des contenus. Cela, certes. autorisait Goldmann à parler de structuralisme, mais bientōt, l'influence grandissante de la linguistique. l'intérêt nouveau envers la réflexion sémiologique, allaient déplacer l'accent vers la productivité du signifiant, les phénomènes narrationnels (l'énonciation), la sémantique structurale. la syntaxe textuelle, sans oublier une conscience plus claire de la nature et du statut du signe en littérature. Tout ce mouvement général depuis une quinzaine d'années a été d'un profit immense pour la sociocritique ${ }^{13}$ même si, à l'heure actuelle, les réalisations, au Québec comme ailleurs, ne se situent pas encore a la hauteur des intentions. Que l'on songe, par exemple, à la fécondité de la notion de code lorsqu'il faut essayer de comprendre la génération sociale du texte, les rapports entre le texte et le hors-texte, le fonctionnement de linstitution littéraire, toutes questions qui concernent ou devraient concerner la sociocritique au premier chef. Pour tirer aujourd'hui les conséquences de la règle méthodologique formulée naguère par Adorno: ‘ La forme a un sens social», la sociocritique ne saurait faire l'économie de l'cuvre comme un ensemble dans lequel les conflits de la société se trouvent traduits ou plutôt transformés selon le jeu des catégories fonctionnelles (et formelles) de tel ou tel discours littéraire.

Sur la question de l'idéologie (laquelle, comme le souligne Claude Duchet. "n'est pas tout le social " ${ }^{14}$ ), il est clair que ces nouvelles perspectives, qui ont commencé à apparaitre quelques années après la publication du Dieu caché. ont entrainé une remise en question du statut traditionnel de l'idéologique dans le texte. Pierre Macherey affirme dès 1966: «[...] les textes littéraires font du langage et de l'idéologie [...] un usage inédit ${ }^{15}$. Dix ans plus tard, France Gaillard donne un nom à cet * usage inédit *: "C'est [...] le processus d'esthétisation qui donne forme à l'idéologie ${ }^{16}{ }^{6}$. Le paradoxe. c'est que la sociocritique qui réclame à la fois une théorie du texte et une théorie sociale, est présentement bien plus a la recherche d'une théorie du texte que d'une théorie sociale. On peut même soutenir que dans la mesure où la sociocritique construira une théorie esthétique du texte répondant à son objet, elle fera avancer du mème coup la théorie sociale. Eu égard à cette dernière, la pensée marxienne a 
presque une valeur méthodologique. Mais il est en train de se produire ceci: ce n'est pas la conception marxiste de la société qui rend compte du texte artistique ; c'est le texte artistique qui travaille et fissure la conception marxiste de la société. Par ailleurs, l'apport de la sociologie de la culture a été et demeure considérable: ainsi, les travaux de Pierre Bourdieu et ceux de Renée Balibar ont été largement lus et commentés dans les universités québécoises.

Je ne me suis écarté qu'en apparence de mon sujet qui est la sociocritique et la littérature québécoise. Il fallait faire un peu état du changement de la conception même du texte depuis Pour une sociologie du roman de Goldmann pour comprendre ce qui se passe actuellement. On trouve dans Etudes francaises. Voix et Images. Lettres québécoises, Études littéraires. Liberté. d'autres revues, bien des articles dont la visée n'est pas sociocritique, qui ressortissent à la narratologie, à la sémantique structurale, mais dont l'importance pour la sociocritique paraît indéniable ${ }^{17}$. C'est qu'au fond, une sociocritique moderne de la littérature québécoise exige pour se constituer une foule de matériaux de provenances diverses.

La relative homogénéité sociale et idéologique de notre société jusqu'à une date toute récente a simultanément favorisé cette sociologisation générale du discours critique que j'ai évoquée plus haut et retardé l'éclosion d'une sociocritique «professionnelle». S'agissant d'une société minoritaire let menacée), la sociocritique se voit, plus qu'ailleurs, guettée par le danger de réitérer LE MEME pour chaque couvre particulière à un moment donné. Ce probleme a d'ailleurs une portée générale: doit-on, synchroniquement, essayer de répéter et décrire ce qu'il y a de commun à tous les textes ou, au contraire, envisager chaque texte comme une réponse différente a la même société? Il me semble que vu les caractéristiques de la culture québécoise, la seconde attitude est préférable.

La notion de culture carnavalesque proposée par Bakhtine ${ }^{18} d^{\prime}$ une part, l'intérêt grandissant que porte, d'autre part, la «nouvelle histoire* envers la culture populaire, les mentalités. la société traditionnelle ${ }^{19}$ ont commencé a changer notre vision du Québec. Plusieurs traits de notre culture, naguère dévalorisés parce que jugés passéistes, anachroniques, liés a des idéaux de droite, se trouvent maintenant l'objet de curiosités d'avant-garde et progressistes. Madeleine foisil, dans la nouvelle revue /'Histoire, écrit avec l'air de s'émerveiller: « La messe c'est, bien sûr, l'assistance à l'office, mais c'est aussi [...] l'occasion privilégiée de la sociabilité paysanne ${ }^{20}$. Quelle découvertel Tout Québécois de plus de vingt-cinq ans sait cela depuis toujours et il l'a d'ailleurs lu au début de Maria Chapdelaine. Effectivement, on voit mal comment une sociocritique sérieuse pourrait éviter de tenir compte des aspects carnavalesques de la culture et de la littérature québécoises.

Ceci posé, on nous permettra de revenir un peu en arrière. La sociocritique au Québec est redevable de beaucoup à Jean-Charles Falardeau. II en fut le premier spécialiste au sens universitaire du terme. Notre société et son roman parut en 196721, Imaginaire social et littérature en 197422. Le travail de Falardeau et les résultats notables qu'il a obtenus illustrent ce fait 
maintes fois observé: quiconque a une bonne connaissance ou une bonne pratique du terrain social ou culturel et de la sensibilité comme liseur peut apporter sa contribution à la sociocritique. Après tout, c'est le cas des plus grands, notamment d'Adorno. A mon avis, ce qu'a fait Adorno pour la musique demeure le modèle inégalé et peut-être inégalable de ce que devrait pouvoir réaliser la sociocritique envers la littérature ${ }^{23}$. Or il est manifeste que la réussite d'Adorno de même que ses attitudes critiques les plus fondamentales sont étroitement liées à sa familiarité profonde, intime, continue avec la culture européenne d'avant la cassure du nazisme, familiarité elle-même inséparable de son appartenance sociale: milieu très cultivé, mère musicienne, lui-même élève d'Alban Berg. etc. II faut garder cette position énonciatrice à l'esprit avant de reprocher à Falardeau sa tendance à lire la société fictive, intra-textuelle du roman québécois comme s'il s'agissait de la société réelle (du moins dans ses textes les plus marquants: “Idéologies et thèmes sociaux dans trois romans canadiens du XIXe siècle», "Le désir du départ dans quelques anciens romans canadiens ", "Les milieux sociaux dans le roman canadien-francais contemporain», a La génération de la relève ${ }^{24}$, $*$ L'évolution du héros dans le roman québécois* 25 . On ne dira pas que Falardeau ignore méthodologiquement les médiations, on dira plutôt qu'il les court-circuite. Les inconvénients découlant de son insistance sur la substance des contenus (les valeurs de la société représentée) sont compensés chez lui par l'ampleur des corpus: il travaille habituellement des ensembles textuels (plusieurs cuvres en synchronie ou en diachronie): les traits observés finissent par avoir le statut dinvariants. C'est la raison pour laquelle ses analyses abondent en observation très justes et très fécondes qui ouvrent la voie à toutes sortes de recherches. Le Romancier fictif ${ }^{26}$, ouvrage que j'ai publié en mil neuf cent quatre-vingt qui porte sur les rapports entre certains phénomènes narrationnels et le statut du langage et de la littérature dans la société québécoise, doit tout à des remarques de Jean-Charles Falardeau sur la récurrence du personnage de l'écrivain dans notre roman.

La question du contenu (par exemple le conténu narratif d'un roman) n'est pas aussi simple qu'il n'y parait. Quand on dit à l'instar de Jean-Louis Cabanès: “C'est la forme même qui signifie l'enracinement idéologique de l'ceuvre ${ }^{27}$, on ne veut pas dire qu'il faut écarter le contenu; on signifie plutôt que le contenu lui-même, comme l'a suggéré Lotman ${ }^{28}$, est subordonné à la réussite littéraire du signifiant, là où tout se joue en réalité. C'est la position de Gilles Marcotte quand il affirme dans le Roman a l'imparfait: "[...] ce que raconte le roman, et la forme dans laquelle il le raconte - qui sont une mème chose, puisque la forme détermine la substance du récit - , sont eux-mêmes justiciables d'une interrogation sociale» ${ }^{29}$. Goldmann disait exactement le contraire: pour lui, telle vision du monde appelle obligatoirement telle ou telle forme. Le Roman a l'imparfait, dans un langage qui emprunte peu en apparence aux perspectives critiques nourries de linguistique et de sémiologie, est en fait un livre très actuel, très moderne, car son objet, c'est la possibilité ou l'impossibilité sociales de certaines formes romanesques au Québec depuis la fin des années cinquante lon pourrait ici remplacer le mot "forme" par celui de "discours" ou de "code") à travers des études sur Bessette, 
Ducharme, Marıe-Claire Blais et Godbout. Jamais la critique marcottienne n'a été aussi vive, déliée. inventive. Et si lẹ Roman à l'imparfait fait date. ce n'est pas tellement parce qu'il résout des problèmes, c'est plutôt parce qu'il pose les bonnes questions, sous l'angle et au niveau qui conviennent aux objectıfs et à la nature de la sociocritique. «[Voyez], par exemple, dit Marcotre, les romans de Mordecai Richler, où la vie montréalaise est décrite avec chaleur et précısion, à travers des événements nombreux et des personnages variés. C'est, en somme, ce que rêvait d'écrire Gérard Bessette dans la Bagarre, et qu'il n'a pas réussi à faire. Mordecai Richler appartient à une culture où l'ordre historique conserve ses pouvoirs: Gérard Bessette. non ${ }^{30}$. La remarquable floraison du roman québécois depuis le début des années soixante donne a voir une grande agilité (et audace) dans l'expérimentation des formes jointe a l'absence - dans le monde représenté par la fiction - de «suivi wistorique, l'absence aussi d'une organisation d'un temps perceptible comme développement humain et social. Quiconque relisait le Roman a limparfait avant le référendum du vingt mai 1980 pouvait prédire la défaite du w oui s et de la liberté. Depuis 1960, nos écrivains ont pourtant nommé, souhaité, magnifié même. le pays indépendant a naître; ils nous montraient du doigt la porte de l'histoire que la volonté du peuple et l'action politique devaient ouvrir. Mais dans leurs romans, «l'ordre historique», pour reprendre les termes de Marcotte, était absent. Marcotte ne veut pas dire ici nécessairement des histoires de pays neufs, de sociétés en voie de développement, d'individus gravissant les échelons sociaux, mais plus simplement et généralement une énonciation selon laquelle le temps joue un rôle important dans la structuration du récit. En fonction de ce temps organisateur, l'avant, l'après, tel moment, tel objet acquièrent du relief et une exactitude obligée, le discours lui-même ne saurait s'accommoder de certaines ruptures. Nous avons mal lu nos écrivains. Ils appelaient le pays libre tandis que la structure mème de leurs couvres en disait l'ajournement ${ }^{31}$.

On aura remarqué que Gilles Marcotte ne sort pas de l'ordre du discours. Comment une sociocritique pourrait-elle v parvenir? Le hors-texte est lui-mème texte. Dans le Romancier fictif ${ }^{32}$, j'ai essayé de préciser, pour le roman québécois d'après 1940, quelques-unes des liaisons discursives entre la société inscrite dans les couvres et la société productrice des couvres. Le Roman a l'imparfait de Gilles Marcotte est le premier exemple d'une sociocritique de la littérature d'ici qui envisage résolument la société elle-mème comme un texte. De ce point de vue. I'ouvrage très documenté de Ben-Zion Shek ${ }^{33}$, Social Realism in the French-Canadian Novel, se situe beaucoup plus nettement dans la perspective de Jean-Charles Falardeau que dans celle de Gilles Marcotte. Shek constate dans les ceuvres des plus importants romanciers depuis le début des années quarante (entre autres Roger Lemelin er Gabrielle Roy) que I'histoire imaginée, la société représentée relatent l'évolution et les conflits de la société réelle. Le roman, ici, cite ou mieux reprend en texte les événements de la société sous la forme d'une sorte d'historiographie fictive. Disons qu'il les scénarise. Pour ma part, à la suite de Gilles Marcotte, je serais enclin à aborder la question autrement : pourquoi, comment écrire à tel moment socio-historique 
donné dans tẹlles conditions concrètes données? Mais bien sür, cela n'enlève en rien son mérite - qui est considérable - à l'ouvrage de B. - Z. Shek.

Deux sociocritiques français se sont intéressés à la littérature québécoise, cet intérèt se limitant toutefois à Marie-Claire Blais. Le premier. c'est Lucien Goldmann lui-même dans ses "Notes sur deux romans de Marie-Claire Clais " ${ }^{34}$. On appréciera de diverses façons une lecture qui semble aggraver - si cela se peut - le sociologisme sans médiations qui caractérisait Pour une sociologie du roman: la grand-mère Antoinette, c'est le monde traditionnel, Emmanuel, le monde moderne industriatisé, etc. Mais Goldmann reconnaît, pour une fois. le type de discours: il s'agit, dit-il, ad'un univers quasi-mythique ${ }^{35}$. C'est ce que ne fait pas curieusement Henri Mitterarid, pourtant linguiste. Il s'étonne du "refoulé» dans Une saison dans la vie d'Emmanuel: "Les silences qui me frappent le plus sont ceux qui gomment toute allusion à l'argent, au régime de la propriété, aux formes économiques du travail agricole et du travail urbain, aux mécanismes de la rémunération, au fonctionnement des pouvoirs»36. Or peut-on sans risques reprocher. comme le fait Mitterand, au discours mythique son absence de réalisme? C'est ici qu'apparaît la connivence profonde, inéluctable - personne n'en est exempt - entre la démarche sociocritique en tant que telle et le roman (et devrait-on ajouter: le roman réaliste). On a souverit reproché a Lukảcs de s'être restreint au réalisme du dix-neuvième siècle. Néanmoins, c'est encore l'attitude aujourd'hui de ce qu'on pourrait appeler l'école de Vincennes (Claude Duchet. Henri Mitterand et leurs collègues): ses travaux portent principalement sur Zola. Flaubert, Stendhal, Balzac. Conclusion: il ne s'agit pas de remettre en question l'approche vincennoise mais de relire Lukács en lui rendant justice.

L'histoire littéraire, entendue au sens habituel, n'a pas sa place ici. $\mathrm{Si}$. l'établissement des textes, le repérage des sources, la recherche biographique demeurent des activités nécessaires, c'est en fonction des besoins de toutes les approches possibles du texte littéraire. Elles constituent la textologie, discipline auxiliaire indispensable ${ }^{37}$. Ce n'est pas le rôle de la sociocritique d'apporter des lumières sur tel événement de la vie d'un auteur, de relever les variantes d'un texte. de préciser sa date de composition, bien qu'elle puisse avoir besoin de toutes ces informations. II convient de ne pas confondre I'histoire littéraire ainsi conçue avec l'histoire de la littérature ou plutôt de l'institution littéraire dont rêvait Barthes et qui aurait été, en fait, une histoire des formes, de la réception des textes, du statut social de la littérature ${ }^{38}$. Cette histoire concerne tellement la sociocritique qu'elle en deviendrait tout un chapitre. Mais elle reste à faire, non seulement au Québec mais ailleurs.

A quels types d'ouvrages sur le Québec lui-même la sociocritique pourrait-elle avoir recours? A mon avis. elle gagne à privilégier les travaux portant sur un aspect ou un autre du discours social, sur la culture, l'idéologie, les mentalités. On retiendra a ce propos l'importante collection «Históire et sociologie de la culture publiée par les Presses de l'Université Laval. II me semble quiun livre comme les Québécois de Marcel Rioux ${ }^{39}$ nous est présentement plus utile que tel travail pourtant fort sérieux sur les classes 
sociales. Dans l'infinie complexité de la superstructure, la sociocritique a besoin, pour s'instaurer, de lancer des passerelles vers tous les autres discours dont l'ensemble compose le discours social.

Voilà donc à larges traits, et dans un langage volontairement simple, le tableau actuel de la sociocritique québécoise. Je n'ai pas voulu aborder les articles car j'aurais alors infligé au lecteur de sèches énumérations, même si quelques titres auraient dû se dégager de l'ensemble comme * La Relève: une idéologie des années 1930 . de Jacques Pelletier ${ }^{40}$, * Le roman québécois: considérations sociologiques» d'Arlette Steiger ${ }^{41}$, ou encore «Aspects du roman québécois des années soixante» de Jacques Michon ${ }^{42}$. $\mathrm{Ce}$ ne sont que trois exemples. J'ai préféré reporter tous ces renseignements dans les indications bibliographiques qui suivent.

1. Eric Auerbach, Mimésis, traduit de l'allemand, Paris, Gallimard, 1968.

2. Georges Lukacs, la Théorie du roman. traduit de l'allemand, Ed. Gonthier. Bibliothèque médiations, 1963.

3. T.W. Adorno, Noten zur Literatur, Francfort, Suhrkamp Verlag, 1958-1965.

4. Mikhail Bakhtine. ICËuvre de François Rabelais et la Culture populaire au Moyen Age et sous la Renaissance. traduit du russe, Paris, Gallimard, 1970.

5. Fernand Quellette, Journal dénoué. Montréal, Presses de l'Université de Montréal, 1974.

6. Pierre V. Zima, Pour une sociologie du texte litteraire, Paris, 10/18, 1978, p. 150.

7. Dans Convergences, Montréal, HMH, 1961.

8. Les Presses de l'Universtté Laval, Québec, 1972.

9. Jean-Paul Sartre. Qu'est-ce que /a litterature?. Paris. Gallimard. 1948, et Questions de méthode. Paris, Gallimard, 1960.

10. Lucien Goldmann, la Dieu caché. Paris, Gallimard, 1959.

11. Presses de I'Université de Montréal, Montréal, 1974.

12. Gallimard, 1964.

13. Je me permets de renvoyer à mon article: «Conditions d'une sociocritique *, Liberté. $\mathrm{n}^{\circ} 111$, mai-juin 1977, pp. 111-117.

14. Claude Duchet, antroduction», la Lecture saciocritique du texte romanesque (sous la direction de Graham Falconer et de Henri Mitterand), Samuel, Stevens. Hakkert, Toronto. 1975, p. X.

15. Pierre Macherey. Pour une théorie de la production litteraire. Paris, Maspero. 1966, p. 66.

16. France Gaillard, Lecture idéologique du texte: Armance de Stendhals, dans la Lecture sociocritique du texte romenesque, op. cit., p. 16.

17. Voir infra des exemples dans les indications bibliographiques.

18. Voir André Belleau, «Bakhtıne et le multiple», Ėtudes françaises, vol. VI, $n^{\circ} 4$. novembre 1970. pp. 481-486.

19. La bibliographie, notamment en France, est déjà très considérable.

20. Madeleine Foisil, a Un gentilhomme campagnard au XVle siècle, le sire de Gouverville*, dans 1 Histoire, n' 2, juin 1978, p. 18.

21. HMH, Montréal. 1967.

22. HMH, Montréal, 1974.

23. Sur l'importance capıtale du modèle adornien, on consultera Frederic Jameson. Marxism and Form, Princeton University Press, 1971, p. 7. pp. 52-53, p. 59, p. 395.

24. Tous dans Notre sactété et son roman, op. cit.

25. Dans /maginaire social et littérature. op. cit. 
26. André Belleau, le Ramancier fictif, essai sur la représentation de l'écrivain dans le roman québécois, Québec, les Presses de l'Université du Québec, 1980.

27. Jean-Louis Cabanès. Critique littéraire et sciences humanes. Toulouse. Privat. 1974, p. 97.

28. louri Lotman, la Structure du texte artistique. tradult du russe, Paris, Gallimard. 1973.

29. Gilles Marcotte, le Roman à l'imparfait, Montréal, La Presse, 1976.

30. Le Roman à l'imparfạt, op. cit., p. 190.

31. Il y a plus d'un texte dans la production abondante de Gilles Marcotte qui intéresse la sociocritique. II convient d'accorder une attention particulière toutefois à ses remarques, dans la Littérature et le Reste, sur les rapports entre l'idéologie de Cite libre et Poussière sur la ville de Langevin (p. 46). sur l'importance des contenus (p. 145), sur l'institution littéraire (p. 183). Voir André Brochu et Gilles Marcotte. La Littérature et le Reste, Les Quinze. Montréal, 1980.

32. Op. cit.

33. Ben-Zion Shek, Social Realism in the French-Canadian Novel. Montreal, Harvest House, 1977 - Voir Joseph Bonenfant: - Social Realism in the French-Canadian Novel de Ben-Zion Shek ou Notre roman entre les faits et la fiction., Voix et Images, IV, 3, avril 1979, pp. 545-547.

34. Dans Structures mentales et création culturelle. Paris, Éditions Anihopos. 1970. pp. 401-414.

35. Ibid., p. 401.

36. Henri Mitterand. «Coup de pistolet dans un concert: Une saison dans la vie d'Emmanuel, Voix et Images, Vol. II, $n^{\circ} 3$, avril 1977, p. 414.

37. De ce qu'il ne faudrait pas hésiter a nommer la littératurologie, le littératurologue étant celui qui, par profession, procède à l'examen spécialisé du discours et des textes littéraires.

38. Roland Barthes, -Histoire ou Littératurex, dans Sur Racine, Paris, Seuil, 1963, pp. 147-167.

39. Marcel Rioux, les Québécois. Paris, Seuil, 1974.

40. Dans Voix et Images du pays $V$. 1972, pp. 69-139.

41. Dans Possibles. Vol. 2, no 1, automne 1977, pp. 81-102.

42. Dans The French Review, Vol. LIII. $n^{\circ} 6$, mai 1980, pp. 812-815.

43. Dans Etudes francaises, $n^{\circ}$ 13/1-2. 1978, pp. 11-34.

44. Editions de I'Université d'Ottawa, 1966.

45. Lire et relire la contribution de Fernand Dumont: "La sociologie comme critique de la littérature=, pp. 225-241. 


\section{INDICATIONS BIBLIOGRAPHIQUES}

On n'a pas cru devoir, à cause de l'objectif du présent *essai», retenir certains articles dont la valeur théorique est considérable mais qui $n$ 'ont pas pour objet principal une ou des ceuvres littéraires québécoises, ainsi "Présupposé. topos, idéologème* de Marc Angenot ${ }^{43}$. Les ouvrages généraux sur la littérature québécoise n'ont pas ici leur place (exemple: le Roman canadien-francais du vingtième siecle de Réjean Robidoux et André Renaud ${ }^{44}$ ). Pour le reste, il s'agit d'un bibliographie sélective; elle ne prétend pas être complète et je m'excuse d'avance des omissions sérieuses.

\section{1) LIVRES (par ordre chronologique)}

En collaboration sous la direction de Fernand Dumont et Jean-Charles Falardeau, Littérature et Société canadienne-française, Québec. Presses de I'Université Laval, $19644^{45}$.

Jean-Charles Falardeau, Notre Sociśté et son roman, Montréal, HMH, 1967.

Axel Maugey: Poésie et Société au Québec (1937-1970). Québec. Les Presses de I'Université Laval, 1972.

Jean-Charles Falardeau, Imaginaire social et littérature, Montréal, HMH, 1974. Gilles Marcotte, le Roman à l'imparfait, Montréal, La Presse, 1976.

Ben-Zion Shek, Social Realism in the French-Canadian Novel, Montreal, Harvest House, 1977.

Robert Major, Parti pris: idéologies et littérature. Montréal, HMH, 1979.

André Belleau, le Romancier fictit, Québec, Presses de I'Université du Québec. 1980.

\section{2) NUMEROS SPECIAUX DE REVUE}

Cahier du C.R.C.C.F., aLangue, littérature, culture au Canada français», Ottawa, 1977.

Études littéraires, aIXE-13, un cas type de roman de masse au Québec», Vol. 12, $n^{\circ} 2$, Québec, août 1979.

Liberté. «L'Institution littéraire québécoise», n 134, Montréal, mars-avril 1981.

Etudes littéraires, «Sémiotique textuelle et histoire littéraire du Québec», Vol. 14, n० 1, avril 1981.

\section{3) ARTICLES (par ordre alphabótique)}

Jacques Allard, «L'idéologie du pays dans le roman québécois contemporain: II n'y a pas de pays sans grand-père et l'intertexte national», Voix et Imoges, Vol. V. n० 1, automne 1979, pp. 117.132.

Jacques Allard, «Les lettres québécoises depuis 1930", University of Toronto Quarterly, n50, novembre 1980.

Bernard Andrès, «Pour une grammaire de l'énonciation pamphlétaire», Études litteraires, Vol. 11, $n^{\circ} 2$, août 1978. pp. 351-372.

Bernard Andrès, "Essai de typologie du discours pamphlétaire québécois», Voix et Images, Vot. 1, n³, avril 1976, pp. 417-431.

Marc Angenot et coll., «Analyse sémiotique d'une chronique de journal: Les Montréalais en parlent». Stratégie, n 5-6, automne 1973.

André Belleau, "Bakhtine et le multiple „, Etudes françaises, Vol. VI, n 4, nov. 1970. pp. $481-487$. 
André Belleau, «La problématique présente de la littérature québécoisen, Liberté, $n^{\circ} 81$, juillet 1972, pp. 13-24.

André Belleau, “Littérature et politique», Stratégie. $n^{\circ}$ 8, printemps 1974, pp. 65-69.

André Belleau, "Ryan, Scully, Victor-Ley Beaulieu: un mème langage de l'immobilité», Liberté, n० 92, mars-avril 1974, pp. 80-87.

André Belleau, "Culture populaire et culture sérieuse dans le roman québécois". Liberté, $n^{\circ} 111$, mai-juin 1977, pp. 31-36.

André Belleau, "Conditions d'une sociocritique», Liberte. $n^{\circ} 111$, mai-juin 1977. pp. 111-117.

André Belleau, "Culture de masse et institution littéraire ", Liberté, $n^{\circ} 120$, nov.-déc. 1978. pp. 3-6.

André Belleau, a Le conflit des codes dans I'institution littéraire québécoise =, Liberté. $n^{\circ} 134$, mars-avril 1981, pp. 15-20.

Roland Bourneuf, * Formes littéraires et réalités sociales dans le roman québécois *. dans Livres et auteurs québécois 1970, pp. 265-269.

Jean-Claude Choul et Michel de Smet, «Des romans bien tranquilles», Voix et Images, Vol. VI, n० 1, automne 1980, pp. 127-145.

Fernand Dumont, *Le temps des aînés *, Etudes trançaises, Vol. 5. n4, nov. 1969. pp. 467.472 .

Marcel Fournier, «La sociologie de la littérature: un discours de célébration », Revue canadienne de sociologie et d'anthropologie, Ed. ACSA. 1976.

Groupe de recherche en sémiotique de I'UOAM, «Pour une segmentation transphrastique du discours littéraire: Sagard en Huronie ». Degrés, $n^{\circ} 4$, été 1978. p. f-f 21 .

J.M. Léard, a Littérature et idéologie», Voix et lmages, Vol. II, n 1, sept. 1976, pp. 92-98.

Gilles Marcotte, «Préface» au Journal de Saint-Denys Garneau. Montréal, Beauchemin, 1954.

Gilles Marcotte, "Notes sur le thème du pays». Voix et Images du Pays. N. 1971. pp. 11-26.

Gilles Marcotte, «Alain et Abel», Cahier du C.R.C.C.F. (Robert Vigneault, éd.), Editions de l'Université d'Ottawa, 1977, pp. 81-89.

Gilles Marcotte, « La problématique du récit dans le roman québécois aujourd hui », Revue des sciences humaines, Université de Lille, $n^{\circ} 1$ 1, 1979, pp. 59-69.

Gilles Marcotte, \& La dialectique de l'ancien et du nouveau chez Marie-Claire Blais, Jacques Ferron et Réjean Ducharmes, Voix et Images. Vol. VI, n 1, 1980. pp. 63-73.

Gilles Marcotte, alnstitution et courants d'air», Liberté, n 134, mars-avril 1981. pp. 5-14.

Jacques Michon, «Aspects du roman québécois des années soixante», The French Review, Vol. LIII, n6, mai 1980, pp. 812-815.

Jacques Michon, «Fonctions et historicité des formes romanesques», Etudes litteraires, Vol. 14, $n^{\circ} 1$, avril 1981, pp. 61-80.

Robert Migner, a Jacques Ferron et I'histoire de la formation sociale québécoise». Etudes francaises. 12/3-4, octobre 1976.

Henri Mitterand, «Coup de pistolet dans un concert: Une saison dans la vie d'Emmanue/s, Voix et Images. Vol. II. $n^{\circ} 3$, avril 1977. pp. 407-417.

Pierre Nepveu, a Le rapport roman-poésie dans la littérature québécoise conternporainen, The French Review, Vol. LIII, n 6, mai 1980. 
Jacques Pelletier, "La relève: une idéologie des années $1930 *$, Voix et Images du pays V. 1972, pp. 69-139.

Jacques Pelletier, a La problématique natıonaliste dans l'œeuvre romanesque de Jacques Godbout „, Voix et Images, Vol. VI, n³. 1981 , pp. 435-452.

Alain Piette. "Un homme et son péché: l'innocente avarice ou te masque idéologique ", Voix et Images, Vol. IV. n 1, septembre 1978.

François Ricard, "André Major ne va pas, il écrit", Liberté, n 109, janvier-février. 1977.

François Ricard, «Ultime réplique à Jacques Pelletier», Liberté, n० 112-113. juillet-octobre 1977, pp. 352-361.

Francois Ricard, Une littérature romantique», Dossier Québec. Paris, Stock, 1979. pp. 177-190.

Fernande Saint-Martin, "La situation de l'art et l'identité québécoisex, Voix et Images, Vol. II, n 1, septembre 1976, pp. 20-27.

Arlette Steiger, a Le roman québécois: considérations sociologiques», Possibles. Vol. 2, n' 1, automne 1977, pp. 81-102.

J.-S. Tassie, a La société à travers le roman canadien-français s, Le Romen canadienfrançais, Archives des Lettres canadiennes, Montréal, Fides, 1971, pp. 153-164.

Georges-Henri Vachon, \& L'espace politique et social dans le roman québécois". Recherches sociographiques, Vol. 7, n 3. sept.-déc. 1966, pp. 259-279.

Georges Vincentier, a L'histoire des idées au Québec - De Lionel Groulx à PaulEmile Borduas», Voix et images, Vol. II, n 1, sept. 1976, pp. 28-46. 